\title{
DIFFICULT INTUBATION IN A PATIENT WITH UNKNOWN SUBGLOTTIC STENOSIS
}

\author{
Santos J., Arede M.J., Freitas M.J., Assunção J.P.
}

Centro Hospitalar Tondela-Viseu

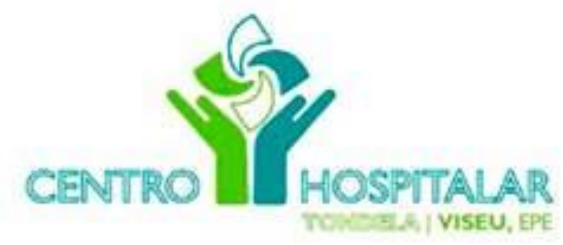

\section{BACKGROUND}

A difficult airway may be defined as the clinical situation in which a trained anesthesiologist experiences difficulties with facemask ventilation, tracheal intubation or both.

Subglottic stenosis is a rare cause for difficult intubation.

- Congenital subglottic stenosis usually presents with varying degrees of dyspnea and stridor.

- Acquired causes include prolonged endotracheal intubation or tracheostomy (90\%), penetrating or blunt neck trauma, chronic inflammatory diseases, gastroesophageal reflux and neoplasm.

- Diagnosis can be confirmed by conventional radiography or CT-scan of the neck.

- Asymptomatic patients are rare and pose a challenge to the anesthesiologist. Stenosis may be suspected during intubation when the tube passes between the vocal cords but resistance is encountered in the stenotic area.

\section{CASE REPORT}

- 46 year old female; admitted for elective vaginal hysterectomy (correction of a complete uterovaginal prolapse).

- ASA II (Obesity [BMI $\left.33,3 \mathrm{~kg} / \mathrm{m}^{2}\right]$ ). No predictive signs for difficult ventilation or intubation. No previous surgeries.

- Monitoring: ASA standard.

- EV induction with fentanyl and propofol. Easy manual ventilation. Grade I laryngoscopy after administration of rocuronium.

- Resistance to progression of the OT tube after the vocal cords - Intubation only possible with a no.4 microlaryngeal tube ( $4^{\text {th }}$ attempt).

- Surgery postponed, extubation without incidents.

- The patient referred previous occasional dysphonia (no dyspnea or dysphagia).

- Otolaryngologic evaluation, fibroscopy and CT-scan: narrowing in an immediately sub-glottic level with an extension of $13 \mathrm{~mm}$ with airway permeability preserved.

- Assumption of a congenital subglottic stenosis.

- The surgery was rescheduled and a sequential anesthesia performed without incidents.
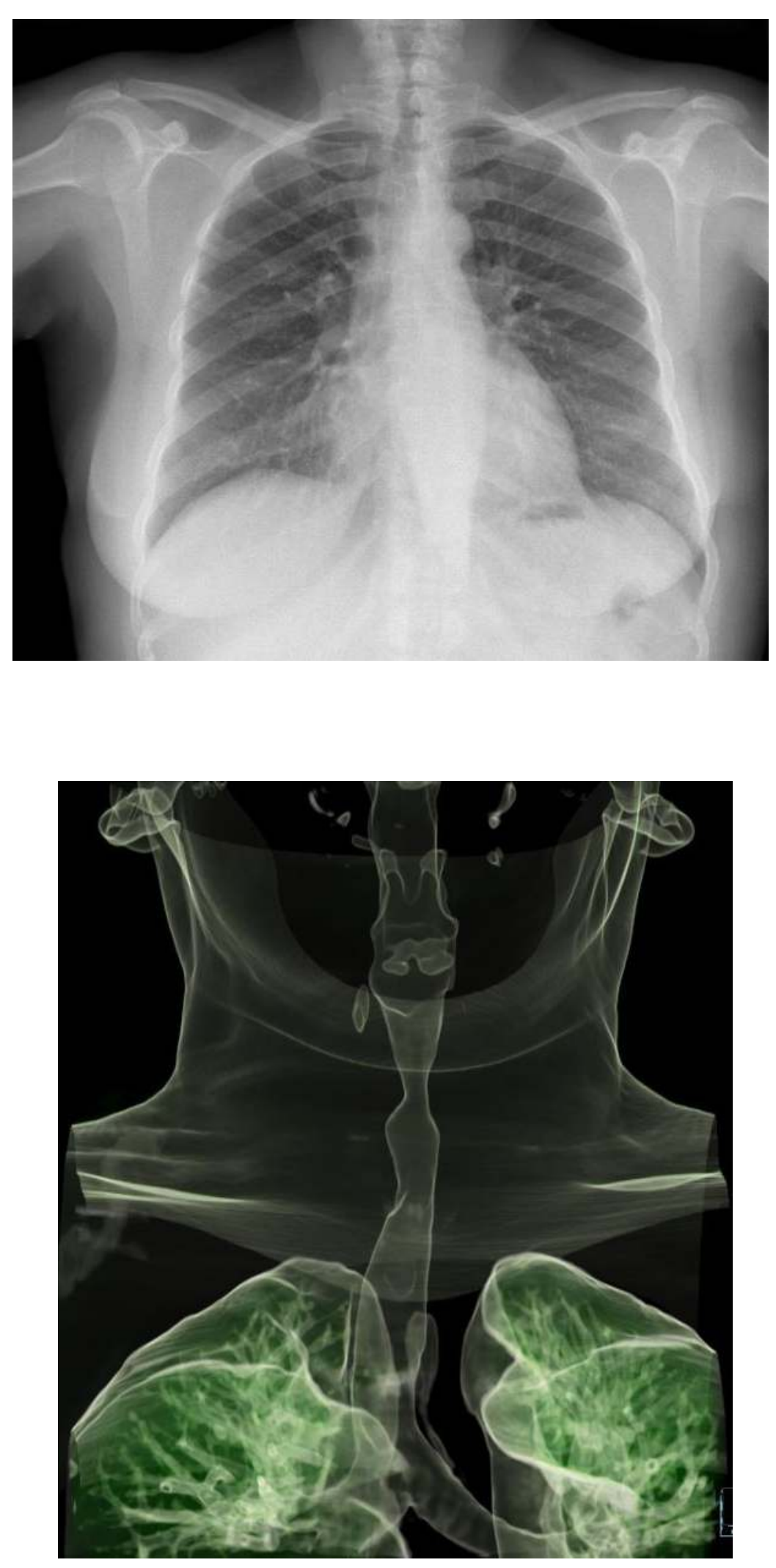

\section{Discussion}

Asymptomatic subglottic stenosis is a rare condition that may present as an unexpected difficult intubation and has high anesthetic risk.

The ability to approach the airway and the type of surgery help the decision on how to proceed - awake the patient and confirm the diagnosis, or approach the airway differently (supraglottic device or invasive surgical airway).

\section{REFERENCES}

Rani N. U. Ramez S. M. Michael, F. Richard. L. F. MD (1995) Acute Management of Unsuspected Subglottic Stenosis by Tracheal Dilation. Anesthesia \& Analgesia Volume 80 Issue 4 - pp 841-843

Low, J. H. Smith, R. The management of laryngeal and subglottic stenosis (2006) Br. J. Anaesth. 96 (6): 803-804.

American Society of Anesthesiologists: Practice guidelines for management of the difficult airway: An updated report. Anesthesiology 2003; 98:1269-1277. 